\title{
A Study on Management of Health Care Infrastructure Development in Rural India: Critical analysis of current status and future challenges
}

Nenavath Sreenu

Assistant Professor in Finance and Accounts, Department of Business Management, Indira Gandhi National Tribal University, Lalpur, Amarkantak, Anuppur (Dist) Madhya Pradesh -484887, India

Correspondence: srinunaikphd@gmail.com

\section{Abstract}

The study has focused on the role of rural health infrastructure development in India. Currently the health infrastructure development of India is poor and it needs fundamental reforms to deal with new emerging challenges. The role of private providers is increasing but simultaneously healthcare facilities are becoming costly. The study surveys the present position of rural health care infrastructure growth, the development of infrastructure, health care facilities, position of human resource, and quality of service delivery.

\section{INTRODUCTION}

The research investigates the rural health infrastructure development in India, one of the largest population's country in the world. Delivery of healthcare facilities to each everyone is a very difficult task. The Indian ministry of healthcare is required to formulate a centralised organisation which would be healthcare centres based, across the 29 states of India, connecting with local self-governance organisations or Panchayats. The public healthcare centres and hospitals have been unsuccessful in developing a proper integrated approach model which can stop the spread of
The paper suggests future challenges of Indian healthcare infrastructure development in rural area, as the burden of disease, financial deficiency in a large section of the population, vaccination policy and poor access to health care. Longevity, literacy and per capita income are further considerations.

Keywords: infrastructure, services, longevity, rural healthcare, delivery and facilities

epidemics and disease. About 70 percent of India's population lives in rural communities but only 20 percent of healthcare centres beds are in rural areas. The paper explores the majorly healthcare infrastructure prerequisite in fast-tracking the jump of healthcare infrastructure both financial and social elements. The healthcare infrastructure development is that which openly simplifies the intervention process. Considerations include transport facility from village to health centres, communication and technology, energy, irrigation, banking and the services facilities comprising of economic development and healthcare infrastructure. [1] 


\section{NEED AND SIGNIFICANCE OF THE STUDY}

The literature review identified the issues in rural healthcare infrastructure service delivery in India. The use of the private sector has increased significantly and, includes increased use by the rural poor suggesting inadequacies and poor access to public healthcare facilities. The bed facilities in the rural healthcare centres have increased from about 36000 to 38000 in the period from 2000 to 2014 , beds in private hospital increased from 49000 to 67500. [2] This represents approximately a $40 \%$ increase in the private hospital bed facilities in a period of 10 years in comparison to approximately $5.5 \%$ increase in the government hospital facilities beds.

About 5000 physicians work in the government healthcare facilities whereas comparatively double the number work in the private sector. The private sector surpassed the government healthcare services facilities in the delivery of refined modalities of diagnosis and therapy, such as CT scan, MRI scan units. Simultaneously, government health services are at risk to internal privatization, having to acquire diagnostic services from the private sector. Emerging nations have been concentrating on pertinent infrastructure improvement in rural healthcare centres, technology development, prevent the disease spread, and healthcare results in terms of deaths and disability-accustomed life years, fundamentally overlooking the service quality improvement characteristic from the patients' perspective.

Research objectives were to examine the policies and practices of government of India for healthcare infrastructure development in rural areas, to investigate the effective competence of the health services facilities and delivery of the infrastructure services to the rural area people of India. To suggest factors which influence the effective management of rural health care infrastructure services hypothesis were constructed to test the data analysis and statistical analysis. These were:

Hypothesis-1: Beneficiaries are satisfied with healthcare infrastructure services provided by the healthcare centres in India.

Hypothesis-2: Beneficiaries are highly dependent on availability of healthcare infrastructure facilities of healthcare centres in India.

Hypothesis-3: There is a substantial deterioration in the rural healthcare centres for the providing operational efficiency of health care system in India.

\section{LITERATURE REVIEW}

Narayana [3] has explained the important objective is to develop the economic feasibility and excellence of health care in government hospital in the states of Andhra Pradesh and Telangana, these two states have introduced a succession of improvements. However, because of an absence of human resource, there has been inactivity and deterioration in the improvement of healthcare centres. Abhijit Banerjee [4] (2004) described health care centres services distribution in the state of Rural Rajasthan. His study reported on a review in Udaipur to measure the delivery of services from healthcare centres to rural people in Rajasthan and the influence it has on the health outcome of the mostly deprived people of the region. He found that the public service is extremely low and that private providers provide the majority of health care provision. 
Chungkham Holendro Singh [5] has explained the PPP model disparity of delivering the infrastructure facilities in health care centres and healthcare budgets in India: The examination discovered that more than 58 percent of the patients have been treated in private healthcare in India. The average cost of treatment in private hospitals is Rs. 5,019 to Rs. 1,307 for government hospitals. The mean accustomed cost of treatment of heart diseases is Rs. 5,981, surveyed by Rs. 5,402, Rs. 4,616, Rs. 2,478 and Rs. 891 for urological diseases, gynaecological disorders, tuberculosis and diarrheal diseases. Shankar Prinja, [6] in 2012 undertook a study to determine inequities in healthcare facilities, service consumption and Out-Of-Pocket (OOP) health expenditures in two States in north India namely, Haryana and Punjab, and Union Territory of Chandigarh. Anitha and Navitha Thimmaih [7] 2013 study focused on the 'Satisfaction from Primary Health Care Services: A Comparative Study of Two Taluks (administrative districts) in Mysore District'. The utilization of any collective services with health care facilities have never been justifiably evaluated. Beside with maximum services facilities provided to the people, it is also significant to be able to relate to admittance to community services particularly health care services and its relationship with other variables. It would be stimulating to study the association between services used and satisfaction.

The World Health Organization [8] report views the access to health workers in isolated and rural health areas as a huge problem of providing connecting points to villages to healthcare centres and the delivery more so than a lack of human resource. The activities of health workers in general, such as income rates, non-attendance, redundancy or twin employment has an association between the causes manipulating the choices and pronouncements of health workers to practice in remote and rural areas and the detractors that could answer to those factors.

Kaveri Gill [9] study concluded that the healthcare National Rural Health Mission (NHRM) is on the right track for the addressing rural health care through the healthcare centres variations inside the healthcare policies. But there are difficulties in execution of the healthcare model, so that provision is variable with respect to healthcare centres infrastructure facilities services, treatments and finance. Whereas with respect to Duggal [10] his study on the availability of health care facilities in India, revealed that India has a plurality of health care systems as well as different systems of medicine. The government and local administrations provide public health care in hospitals and clinics. The rural health centres are underutilized because they cannot make available services their customers anticipated in quantity of consideration and medication and services. Hanan AL-Ahmadi et al [11] found that the factors that are determining the high-quality care are management \& administrative aspects, execution of suggestion-constructed practices, specialized expansion, use of recommendations to subordinate care and structural values. The other factors that are required in order to improve quality are the knowledge and skills of staff.

The rural health care system in India has been established as a three tier Model with Sub Centre, Primary Health Centre (PHC) and Community Health Centre $(\mathrm{CHC})$ being the important three pillars for rural healthcare development. The Sub Centre is the 
furthermost marginal and main connecting point between PHCs and $\mathrm{CHCs}$, however the $\mathrm{PHC}$ is the first connecting centre to the village and the medical centres in the rural area, and $\mathrm{CHC}$ is the centre for four PHCs, and provides facilities for the beneficiaries. The development of these health infrastructure facilities, especially growth of the Sub Centres is a precondition for the whole progress of the complete system. Accessibility of manpower and quality of health care services are other significant components of health care infrastructure.

The founding of these rural centres is constructed on definite population norms which is different for Plain areas and Hilly areas according to the NRHM [12]. "The population norm in Plain areas is $\mathbf{5 0 0 0}$ per Sub Centre, 30000 per PHC and 120000 per CHC; whereas that for Hilly/Tribal/Desert areas is $\mathbf{3 0 0 0}$ per Sub Centres, 20000 per PHC and 80000 per $\mathrm{CHC}$. Further, there will be six Sub Centres per $\mathrm{PHC}$ and four PHCs per CHC. The population norm for a female health worker at Sub Centre \& PHC and a male health worker at Sub Centre are fixed at 5000 for Plain areas and 3000 for Hilly/Tribal/Desert areas". [12]

\section{METHODOLOGY}

The research study has collected the information from primary data and secondary data. The first-hand data has been collected from the beneficiaries and provider in the rural healthcare centres by administering structured questionnaire. The questionnaire has been a blend of open ended and close-ended questions. An appropriate scaling technique has been used to measure the response and all secondary data pertinent document and published reports have been referred and the researcher has visited the field to collect the information. Semi structured interviews were conducted with provider of rural healthcare services such as medical officer, store assistant and ASHA (Accredited social health activists) workers in selected healthcare centres.

The research paper followed mixed methods and analytic tools have been used to assess the healthcare infrastructure facilities from the rural Healthcare centres in the form of both structured and semi-structured questions from the different stakeholders and providers as well as attention groups discussion planned to highpoint the foremost extents of disappointment of both facilities providers (health workers) and their clients. The main determination is to understand the variety in the plaintiff's responses [13] The total sample size is 450 based on random sampling methods. The data has been collected from the rural healthcare centres from the providers, beneficiaries and NGOs.

\section{DATA ANALYSIS}

The data analysis involved a multiple regression model used for analysing variables (total 10 variables). The detailed of the data analysis has been made available but not been included because of Journal word limit requirements.

\section{RESULTS AND DISCUSSION}

The results indicate infrastructure facilities improvement of the rural healthcare centres in India were found to be insignificant and that the PHCs infrastructure facilities are in very poor condition and, it needs to improve the facilities in the PHCs of the tribal area region. Most of the beneficiaries and providers tend toward strongly agreeing with the concept that the rural healthcare infrastructure has not significantly affected healthcare delivery services in the state of the Andhra Pradesh, Telangana, Madhya Pradesh, CG, UP and Orissa. The research study has concluded that 
the rural health care infrastructure facilities have not significantly affected healthcare delivery services in India. A comparison of common factors clearly shows that infrastructure delivery services at PHCs and availability of critical manpower at PHCs emerge as key factors for the effective delivery of services in the rural area of India showing the improvement and evaluation of the performance of the rural area health centres.

The study has taken into consideration three ways of services connected to rural healthcare delivery facilities, one is infrastructure improvement at PHCs, the availability of critical manpower at PHCs and coordination with community by Accredited social health activists (ASHA) at PHCs.

\section{CONCLUSION}

Healthcare infrastructure improvement is a vital aspect in the rural areas of India. The research paper analysed various factors that contribute to the performance of rural healthcare infrastructures. It can be concluded that reliability in healthcare centres delivery, can be improved by providing infrastructure facilities, involvement of and coordination with the community as influencing the performance of healthcare services. The study also identified the factors influencing healthcare services delivery and development with infrastructure facilities and concluded that lack of effective delivery services in rural services centres.

The majority of the respondents have expressed their view in respect of poor facilities in rural healthcare centres, those shortages in energetic human resource facilities in healthcare centres and very poor management in managing the levels of supporting staff essential. This has been the main antagonistic effect on the rural healthcare centres services and the insignificant impact on the health-care infrastructure delivery services.

\section{Reference}

1. Bhandari L, Dutta S. India Infrastructure Report; 2007. Health infrastructure in rural India

2. Kunhikannan TP, Aravindan KP. 2000. Changes in the health status of Kerala: 1987-1997. Discussion Paper No. 20: 26-36. Thiruvananthapuram: Centre for Development Studies.

3. Narayana, KV (2003): Changing Health Care System. Economic \& Political Weekly. March 22-29, 2003. P. 1230-1241.

4. Abhijit Banerjee, et al (2004): Health Care Delivery in Rural Rajasthan. Economic \& Political Weekly. February 28, 2004. P. 944-949.

5. Chungkham Holendro Singh (2009): The Public-Private Differential in Health Care and Health-Care Costs in India: The Case of In-patients. Journal of Public Health. Vol. 17. 2009. P. 401-407.
6. Shankar Prinja, et al (2013): Equity in hospital service utilisation in India. Economic \& political weekly. Vol. 48. No. 12. March 23, 2013. P.52-58

7. Anitha, CV and Navitha Thimmaiah (2013):

Satisfaction from Primary Health Care Services: A Comparative Study of Two Taluks in Mysore District. PARIPEX: Indian Journal of Research. Vol. 2. No. 12. December 2013. P. 45-49.

8. World Health Organisation 2009, Increasing access to health workers in remote and rural areas through improved retention, Background paper for the first meeting to develop evidence-based recommendations to increase access to health workers Geneva, 2009. 9. Kaveri Gill, A Primary Evaluation of Service Delivery under the National Rural Health Mission (NRHM): Findings from a study in Andhra Pradesh, Uttar Pradesh, 
Bihar and Rajasthan, Working Paper 1/2009-PEO,

Planning Commission of India, 2009.

10.Duggal $R$, Health care utilization in India, Health for the Millions 1994; 2(1): 10-2.

11. Hanan ALAhmadi, Martin Roland, and Quality of primary health care in Saudi Arabia: a comprehensive view, International Journal for Quality in Health Care 2005;17(4): 331-46

12.National Rural Healthcare Mission (NRHM):

Evaluation Report. Published by Planning Commission,

Government of India. February 2011 